\title{
Comunicación
}

\section{Un caso de hidrometra en una perra mestiza}

\author{
A case of hydrometra in a crossbred bitch
}

Rodolfo Dextre T., ${ }^{1,3}$, Jacqueline Cahua U., Nieves Sandoval C. ${ }^{2}$

\section{Resumen}

\begin{abstract}
El presente estudio reporta un caso de hidrometra en un canino hembra de 12 años que fue llevada a la Clínica de Animales Menores de la Facultad de Medicina Veterinaria de la Universidad Nacional Mayor de San Marcos (Lima, Perú). La perra presentaba signos clínicos de gestación aparente de 45 días, pero sin historia de celo o monta. Las constantes vitales, uroanálisis, hematología y bioquímica sanguínea indicaron valores dentro de los parámetros normales. En el examen ecográfico se observaron signos compatibles con hidrometra. Se llevó a cabo una ovariohisterectomía y se tomaron muestras uterinas para histopatología, lo cual confirmó el diagnóstico; además de una hiperplasia endometrial quística concomitante en uno de los cuernos uterinos.
\end{abstract}

Palabras clave: hidrometra; Canis lupus familiaris; hiperplasia endometrial quística; ecografía

\section{Abstract}

The present study reports a case of hydrometra in a female canine of 12 years who was taken to the Small Animals Clinic of the Faculty of Veterinary Medicine of the Universidad Nacional Mayor de San Marcos (Lima, Peru). The bitch had clinical signs of apparent gestation of 45 days, but no history of oestrus or mounts. Vital signs, uro-analysis, haematology, and blood biochemistry indicated values within normal parameters. The

${ }^{1}$ Clínica de Animales Menores, Facultad de Medicina Veterinaria, Universidad Nacional Mayor de San Marcos, Lima, Perú

${ }^{2}$ Laboratorio de Histología, Embriología y Patología Animal, Facultad de Medicina Veterinaria, Universidad Nacional Mayor de San Marcos, Lima, Perú

${ }^{3}$ E-mail: rodolfodextretorres@gmail.com

Recibido: 11 de abril de 2017

Aceptado para publicación: 15 de septiembre de 2017 
ultrasound examination showed compatible signs with hydrometra. An ovariohysterectomy was performed, and uterine samples were taken for histopathology, which confirmed the diagnosis, in addition to a concomitant cystic endometrial hyperplasia in one of the uterine horns.

Key words: hydrometra; Canis lupus familiaris; cystic endometrial hyperplasia; echography

\section{INTRODUCCIÓN}

La hidrometra es una patología cuyo nombre literalmente significa «agua» o líquido en el útero. Smith (2010) la define como la acumulación de líquido casi translucido en el útero. La incidencia de patologías como la hidrometra en perras es entre 0 y $20 \%$ (Galina y Valencia, 2009), y mayormente en perras de edad madura, después de un periodo de celo y secundario o concomitante a una hiperplasia quística endometrial (HEQ). Dentro de los diagnósticos diferenciales al examen físico se encuentran la mucometra, hemometra y piometra (Martí, 2011).

\section{Caso Clínico}

Paciente canino, hembra, de raza mestiza, con $5 \mathrm{~kg}$ de peso y de 12 años aproximadamente. La perra fue recogida de la calle siete años atrás.

El propietario llevó a la paciente a la clínica de la Facultad de Medicina Veterinaria de la Universidad Nacional Mayor de San Marcos (Lima, Perú), debido a que presentaba el abdomen abultado, el cual había crecido en forma notable durante el último mes, de allí que sospechaba de gestación. Sin embargo, no había observado signos de sangrado por celo, pero en ciertas ocasiones notó que un macho, que también lo tenía como mascota, la había cortejado en forma eventual, aunque sin evidencia de monta. La perra no había recibido hormonas ni otros medicamentos, como corticoides. Asimismo, estaba inmunizada con las vacunas recomendadas para su edad y había recibido los tratamientos antiparasitarios correspondientes. En los antecedentes se mencionó que había tenido una secreción vulvar blanquecina, pero al examen clínico no se encontró evidencia alguna. Tenía apetito y comportamiento normal.

Al examen clínico, tenía temperatura normal, buen estado deánimo, condición corporal de 4/5. A la palpación, el abdomen se presentó muy distendido, tenso y pendulante. Se realizaron pruebas complementarias como uroanálisis, hematología y bioquímica sanguínea, encontrándose los valores dentro de los parámetros normales.

El estudio ecográfico evidenció los cuernos uterinos distendidos, con colección líquida anecoica abundante, de $50 \mathrm{~mm}$ de diámetro transverso y paredes de $2.2 \mathrm{~mm}$ de espesor (Figura 1). Se le diagnosticó como el complejo hidrometra, mucometra o piometra.

Se le practicó a la perra una ovariohisterectomía, no habiendo contratiempos en la cirugía ni en el posoperatorio. En la cirugía se encontró el cuerno izquierdo dilatado, lleno de líquido y de paredes delgadas, casi traslúcidas. El cuerno uterino derecho tenía características similares, pero parte del cuerno estaba endurecido, de paredes gruesas, reducido en su luz, no dilatado y sin contenido luminar (Figura 2).

Se evaluaron cortes de tejido del cuerno uterino fijados en formol y coloreados con hematoxilina y eosina (H-E) para el estudio histopatológico. Se observaron múltiples quis- 


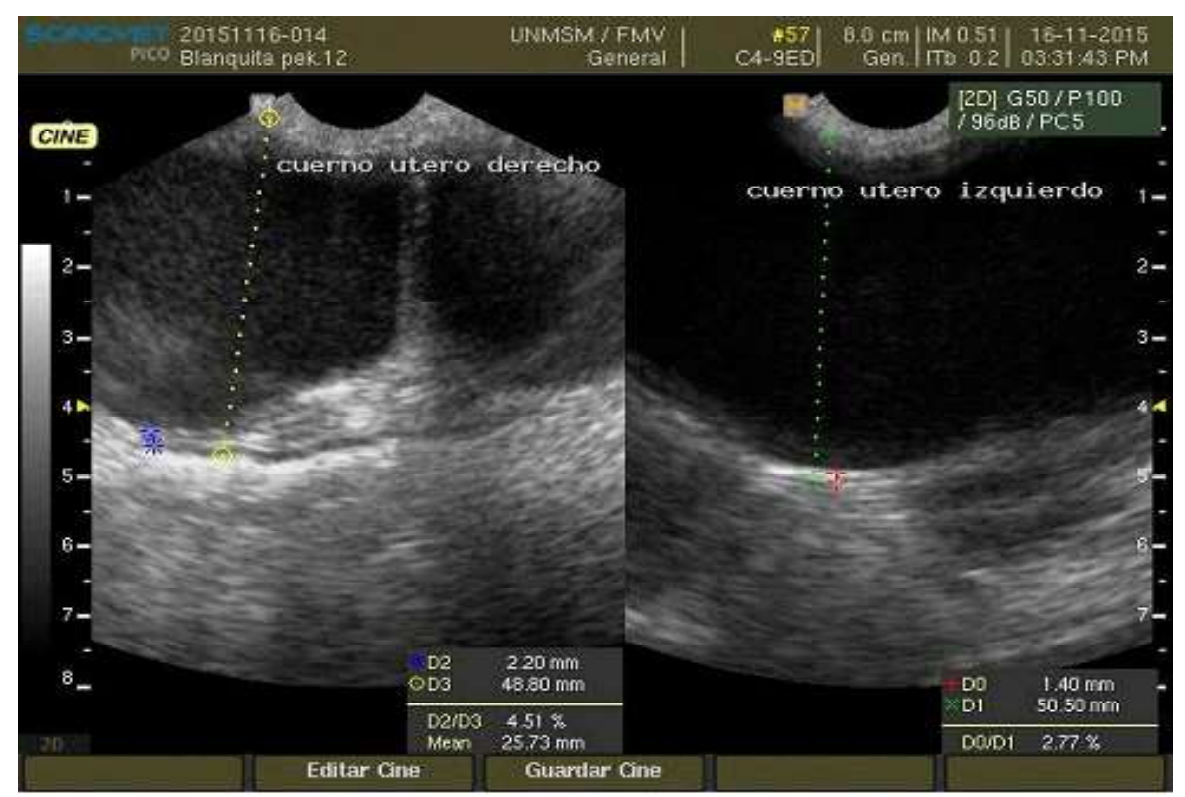

(a)

(b)

Figura 1. Imágenes ecográficas de una perra mestiza de 12 años. (a) Se observa parte del cuerno uterino derecho dilatado con abundante contenido anecoico. (b) Se observa el cuerno uterino izquierdo totalmente dilatado y con similares características, llegando a un grosor de $50.5 \mathrm{~mm}$

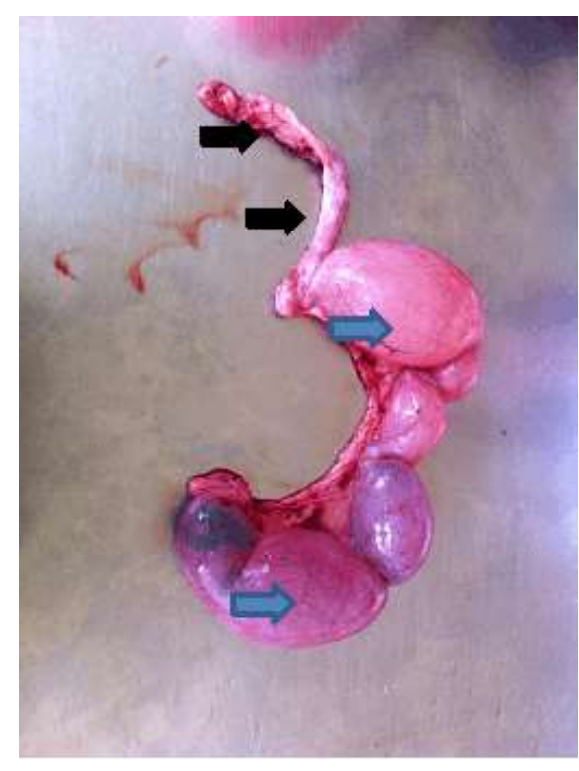

Figura 2. Tracto reproductivo de perra mestiza de 12 años mostrando el cuerno izquierdo distendido con colección líquida acuosa, y parte del cuerno derecho hiperplásico. Probable hidrometra (flecha azul). Posible hiperplasia endometrial quística (HEQ) (flecha negra) tes endometriales de tamaño diverso, con presencia de un fluido translúcido, sin presencia de células inflamatorias. El epitelio de las glándulas uterinas en los acinos cambió de columnar a cúbico y plano, evidenciando atrofia de la mucosa. En otros acinos glandulares se observó incremento en el número de células, dando la apariencia de estratos (Figura 3).

Con base a las lesiones mencionadas se diagnosticó como una hiperplasia endometrial quística con hidrometra, confirmando las observaciones macroscópicas.

\section{Discusión}

La hidrometra es también conocida como una falsa gestación donde se acumulan líquidos asépticos en la luz uterina, sin que exista gestación real, pero en presencia de un cuerpo lúteo (Feldman y Nelson, 2000; Matthews, 2002). El tratamiento médico pue- 


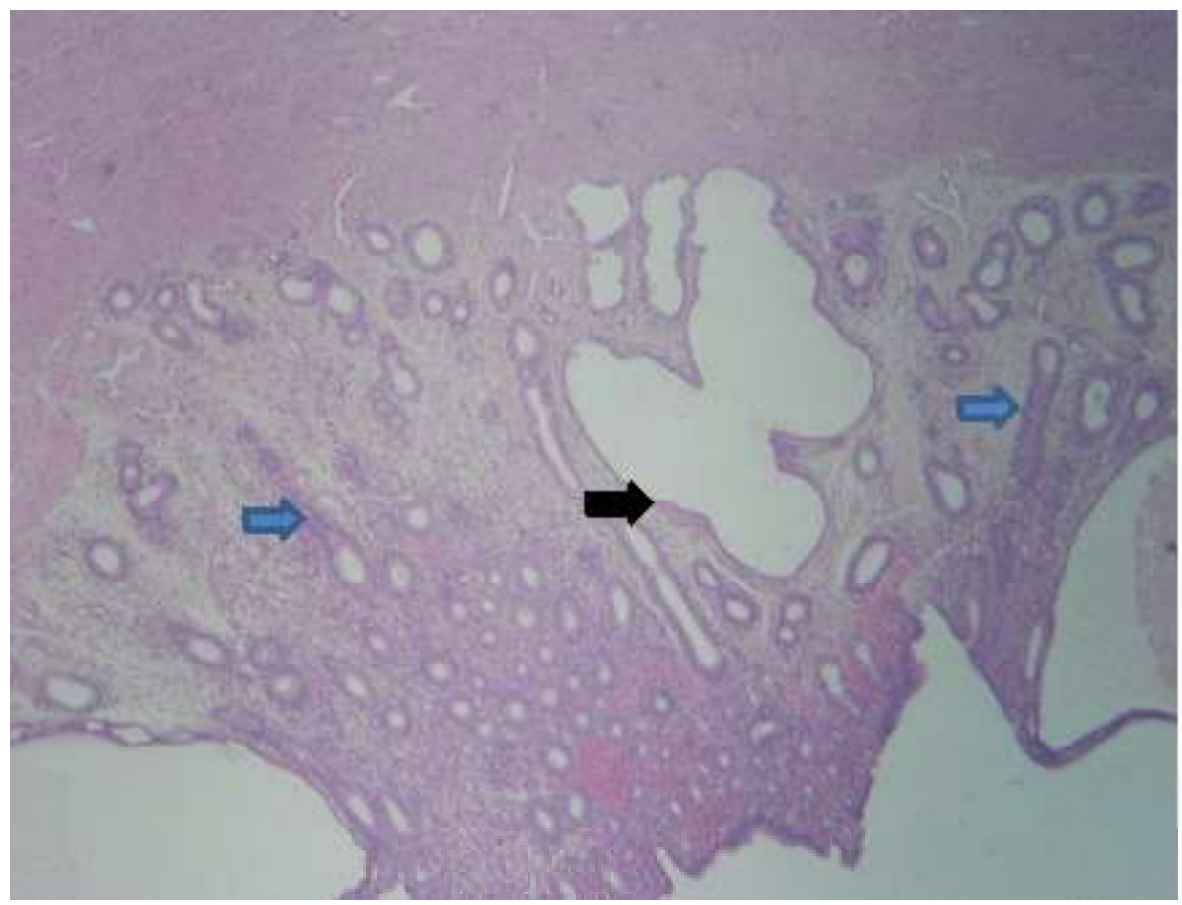

Figura 3. Corte histológico del cuerno uterino de una perra mestiza de 12 años. Se observan alteraciones de las estructuras epiteliales de los acinos. Epitelio cúbico atrófico (flecha negra); epitelio hiperplásico (flechas azules)

de provocar la rotura uterina o la salida del contenido al interior de la cavidad abdominal (Bonagura y Twedt, 2010), de allí que el tratamiento de elección con perras con el cuello del útero cerrado es la ovariohisterectomia completa, tratamiento que se recomendó en este caso.

La referencia del dueño sobre la falta de interés de la perra con el macho era concordante con el caso de la hidrometra, ya que este proceso es considerado como causa de anestro (Matthews, 2002). Por otro lado, la HEQ, patología que fue observada tanto macroscópicamente como microscópicamente, puede llegar a dificultar la concepción (Hagman et al., 2006), cambio adaptativo que presentó la mascota macroscópicamente y fue corroborado por el estudio microscópico.
Una de las causas de la hidrometra y hematometra podría ser que, durante el diestro, la progesterona produce un incremento de la actividad de las glándulas secretoras endometriales, engruesa el endometrio y disminuye la contractilidad miometrial, pudiendo causar el cierre funcional de la cérvix y de la inhibición del drenaje de los exudados uterinos. Estos efectos se acumularían después de varios ciclos, lo que explica la mayor incidencia en hembras de mediana y avanzada edad (SEVC, 2009; Kustritz, 2010); lo cual concuerda, asimismo, con la edad de la mascota en estudio.

Es difícil diferenciar clínicamente una piometra de una hidrometra o mucometra (Hagman et al., 2006). No obstante, la primera requiere de tratamiento urgente ya que puede ser letal, en tanto que las dos últimas no constituyen una urgencia veterinaria. 
Los diagnósticos histopatológicos y ecográficos concordaron, siendo este último el más útil y sensible para lograr un diagnóstico definitivo, ya que esta patología no presenta signos sistémicos significativos (Kustritz, 2010; Martí, 2011). En cabras, sin embargo, se ha detectado acumulación de líquido turbio en grandes compartimentos separados por paredes tisulares ondulantes (Smith, 2010).

La hidrometra se presenta usualmente acompañada de la HEQ, cambios que fueron observados en el útero de la paciente, tanto macroscópicamente como por histopatología. Este último confirma el diagnóstico de HEQ; sin embargo, otros medios diagnósticos no invasivos que pueden ser utilizados son la ecografía, radiografía y palpación clínica (Bonagura y Twedt, 2010; Couto y Nelson, 2010; Smith, 2010).

El examen citológico de secreciones vaginales es de gran utilidad en los primeros momentos, ya que permite diferenciar entre piometra, mucometra, hidrometra y hematometra (Martí, 2011). En este caso, la mascota tuvo secreción vaginal días antes de que la trajeran a la clínica, pero no presentaba secreción alguna en el momento del examen clínico. Actualmente, se puede usar métodos de análisis como el PG-metabolite, con una sensibilidad de $98.3 \%$ y especificidad de $80 \%$ para diferenciar entre una piometra y una $\mathrm{HEQ} /$ mucometra en perras cuando se dispone de un diagnóstico de útero con conteniendo líquido (Hagman et al., 2006).

La etiología exacta de esta enfermedad se desconoce, pero se le ha asociado a diversos agentes infecciosos y a ciertos tratamientos hormonales (Galina y Valencia, 2009), y en otras especies animales a fitoestrógenos (Smith, 2010).

\section{Literatura Citada}

1. Bonagura J, Twedt D. 2010. Kirk, Terapéutica veterinaria actual. $14^{\circ} \mathrm{ed}$. España: Elsevier. 1388 p.

2. Couto G, Nelson R. 2010. Medicina interna de pequeños animales. $4^{\circ}$ ed. España: Elsevier. 1467 p.

3. Feldman E, Nelson R. 2000. Endocrinología y reproducción en perros y gatos. $2^{\circ}$ ed. México: McGraw-Hill. 621 p.

4. Galina C, Valencia J. 2009. Reproducción de animales domésticos. $3^{\circ}$ ed. México: Limusa. 582 p.

5. Hagman R, Kindall H, Fransson BA, Bergström A, Holst BS, Lagerstedt AS. 2006. Differentiation between pyometra and cystic endometrial hyperplasia/ mucometra in bitches by prostaglandin $\mathrm{F}_{2 \alpha}$, metabolite analysis. Theriogenology 66: 198-206. doi: 10.1016/j.theriogenology.2005.11.002

6. Kustritz M. 2010. Clinical canine and feline reproduction. USA: WileyBlackwell. 316 p.

7. Martí S. 2011. Reproducción y neonatología canina y felina. España: Servet Editorial.

8. Matthews J. 2002. Enfermedades de la cabra. $2^{\circ}$ ed. España: Ed Acribia. 397 p.

9. Smith B. 2010. Medicina interna de grandes animales. $4^{\circ}$ ed. España: Elsevier Mosby. $1813 \mathrm{p}$.

10. [SEVC] Southern European Veterinary Conference. 2009. Barcelona: Aspectos clínicos de la patología uterina. [Internet]. Disponible en: http:// www.rednacionaldeveterinarias.com.uy/ articulos/anestesiologia/patologia_uterina.pdf 